\title{
Cardiac rehabilitation in chronic heart failure
}

\author{
Romualdo Belardinelli \\ Cardiologia Riabilitativa "GM Lancisi”, Azienda Ospedaliera Universitaria Ospedali Riuniti Ancona, Italy
}

\begin{abstract}
Chronic heart failure is a complex clinical syndrome characterized by multi-organ dysfunction and progressive functional deterioration. From the result of the literature, it is evident that the optimal care for heart failure is far from being reached, and exercise-based cardiac rehabilitation (Ex-CR) is a multidisciplinary therapeutic option with important clinical benefits. Longitudinal controlled studies and meta-analyses have demonstrated that Ex-CR improves functional capacity by $12-25 \%$, and improves outcome by significantly decreasing hospital readmissions and cardiac mortality. These results have been obtained in patients with systolic dysfunction (HF-REF) with moderate aerobic endurance programs. More recently, greater improvements in functional capacity have been obtained even in older patients ( $>75$ years) with high intensity interval training, but the number of patients is too small at present to reach definitive conclusion, and follow up is lacking. In diastolic heart failure (HF-PEF) improvements in functional capacity have been described in a total of 282 patients, with similar results as HF-REF but no evidence on outcome. Ex-CR programs are safe with a very low number of cardiac arrest (1/300,000 patient/hour) in different trials. Clinical benefits are the result of central and peripheral adaptations induced by exercise, which acts as a trigger of protein synthesis by specific genes activated by it. It is crucial to maintain a specific stimulus by repeating exercise bouts at least 2-3 times per week all life long, because after 2-3 weeks of inactivity functional adaptations disappear.
\end{abstract}

Corresponding author: Romualdo Belardinelli, Cardiologia Riabilitativa "GM Lancisi", Azienda Ospedaliera Universitaria Ospedali di Ancona, Via Conca 71, Torrette (AN), Italy.

Tel. +39.071.5965798 - Fax +39.071.5965042.

E-mail: romualdo.belardinelli@ospedaliriuniti.marche.it

Key words: Cardiac rehabilitation; chronic heart failure; diastolic heart failure.

Received for publication: 02 April 2016

Accepted for publication: 29 June 2016

(C) Copyright R. Belardinelli, 2016

Tipografia PI-ME Editrice, Italy

Monaldi Archives for Chest Disease Cardiac Series 2016; 86:753

doi: 10.4081/monaldi.2016.753

This article is distributed under the terms of the Creative Commons Attribution Noncommercial License (by-nc 4.0) which permits any noncommercial use, distribution, and reproduction in any medium, provided the original author(s) and source are credited.

\section{Introduction}

Chronic heart failure (CHF) is a complex clinical syndrome characterized by multi-organ dysfunction and progressive functional deterioration. Every year, CHF has been diagnosed in 1 to $5 / 1000$ subjects. Its prevalence and incidence increases with age, with a 5 -year mortality rate of $45 \%$ [1-3]. The most frequent causes of CHF are coronary artery disease and hypertension, which account for $80 \%$ of all causes. Despite the progress of medical therapy over the last decades, which has been effective in reducing mortality rate in the hospital, the prognosis of CHF is unchanged, with $30-40 \%$ of patients dying within one year from diagnosis, while outpatient clinics increased the volume of activity and costs of care. From the result of the literature, it is evident that the optimal care for heart failure is far from being reached, and there is a need to find new methods of treatment in order to improve functional capacity, quality of life and clinical outcome [4-6].

Over the last three decades, exercise-based cardiac rehabilitation (ExCR) moved from a research tool to a clinical well-defined therapeutic option in patients with CHF. The European Society of Cardiology and the American Cardiology Societies (AHA/ACC) both recommend ExCR in stable CHF patients on the basis of previous longitudinal trials and more recent meta-analyses [7-9].

\section{Physiopathology}

A reduced exercise tolerance is common in CHF as a consequence of a vicious cycle of cardiocirculatory failure and deconditioning. Heart failure stimulates adrenergic activity and renin-angiotensin system, and is a major determinant of skeletal muscle atrophy, which decreases aerobic enzymes activity favoring exercise intolerance [10]. This cascade of negative events is similar between diastolic heart failure (HF$\mathrm{PEF}$ ) and systolic heart failure (HF-REF), suggesting that ejection fraction is unrelated to peripheral abnormalities, which seem to play a major role in exercise deconditioning and clinical deterioration [11].

\section{Exercise training}

Exercise training has been considered as the key point of cardiac rehabilitation programs since 1985 . From the scientific evidence of the Duke group in the US and the Brompton's group in UK, other trials were performed in western countries over the next 2 decades, demonstrating the efficacy of exercise-based cardiac rehabilitation in HF-REF patients first, and HF-PEF later on [12-20].

\section{HF-REF}

The ExTraMATCH meta-analysis summarized the results of the first group of trials on 801 HF-REF patients randomized into two groups: 
exercise ( $n=395)$ and controls $(n=406)$ [9]. Exercise training was performed on top of medical therapy at moderate intensity $(60-80 \%$ of peak V02), under supervision for 213(135) days with a mean follow up of 705(729) days. Functional capacity was improved, ranging 12-25\%. Overall, there were 88 deaths in the exercise arm (median time to event, 618 days) and 105 in the control arm (421 days). Mortality was significantly lower in the exercise group (log rank $\left.\mathrm{X}^{2}=5.9, \mathrm{p}=0.015\right)$ and the hazard ratio for mortality was 0.65 ( $95 \%$ confidence interval 0.46 to 0.92 ). These results would imply 17 treated patients to prevent one death in two years.

HF-ACTION was a multicenter, randomized controlled trial designed to measure the effects of exercise training in stable HF-REF patients ( $n=2331$ ) on functional capacity, quality of life measures of outcome such as mortality and re-hospitalization [21]. Patients were randomized to 36 sessions of supervised aerobic endurance exercise at $60-70 \%$ heart rate reserve followed by home-based training or usual care. At a median follow-up of 30 months, there was a $7 \%$ reduction in the primary combined end point ( $\mathrm{p}=0.13)$. However, after adjustment for prespecified predictors of mortality, such as duration of exercise test, LVEF, BDI score and history of atrial fibrillation, there was a statistically significant reduction in mortality (HR $0.89, \mathrm{p}=0.03$ ). The improvement in peak $\mathrm{VO}_{2}$ was only $4 \%$, probably due to biases such as low adherence to training in the training group and regular home exercise in many control patients. In a subsequent analysis, patients who exercised as prescribed experienced a $>30 \%$ reduction in mortality or hospitalization. For every $6 \%$ increase in $\mathrm{VO}_{2}$ peak there was a $5 \%$ lower risk of the primary end point $(\mathrm{p}<0.001)$ and an associated $8 \%$ lower risk of combined cardiovascular mortality and hospitalizations $(\mathrm{p}<0.001)$.

In order to improve long-term outcome, adherence to exercise prescription is crucial. This concept has been demonstrated in a recent paper where $123 \mathrm{HF}-\mathrm{REF}$ patients followed a supervised program of exercise training at $60 \%$ of $\mathrm{VO}_{2}$ peak twice a week for 10 years in a coronary club with periodic checks in the hospital [22]. The $\mathrm{VO}_{2}$ peak was maintained at more than $60 \%$ throughout the 10 years [average: 65.3 (3\%)], whereas it was less than $55 \%$ in untrained patients [52 (8\%), $\mathrm{p}<0.01]$. The training group had a better quality of life $(\mathrm{p}<0.05)$, fewer hospital readmissions $(\mathrm{p}<0.001)$, and a lower cardiac mortality $(\mathrm{p}<0.001)$ than the control group. Authors concluded that supervision seems to be a crucial factor to reduce dropouts and increase adherence to long-term exercise sessions. The coronary club may be an efficient model to apply in cardiac rehabilitation in future studies.

\section{HR-PEF}

The results of 7 controlled trials on HF-PEF patients have demonstrated that exercise training improves aerobic capacity and quality of life at similar amounts of endurance training in HR-REF patients. A total of 282 patients were studied, 157 underwent endurance exercise training at moderate intensity, three times a week for 12-52 weeks [2329]. In the only randomized multicenter study, the Ex-DHF pilot study (Exercise Training in Diastolic Heart Failure), $\mathrm{VO}_{2}$ peak increased by $16 \%$ ( $\mathrm{p}<0.001$ vs controls) and was correlated with diastolic filling $\mathrm{E} / \mathrm{e}$ ' change $(\mathrm{r}=-0.37, \mathrm{P}=0.002)$ and SF-36 physical functioning $(\mathrm{r}=-0.46$, $\mathrm{p}<0.001)$. At present, there is not sufficient evidence that Ex-CR has positive effects on the clinical outcome of HF-PEF patients.

\section{Patient selection}

The condition sine qua non to obtain benefits from exercise training in CHF is clinical stability. In all studies, patients enrolled did not have severe ventricular arrhythmias, unstable angina and signs or symptoms of worsening heart failure over the last 3 months. Mean age was 59 years in a recent review (range 43-72 years) [14]. The majority of patients are men, NYHA class II and III, ischemic etiology. The dose of exercise training across studies ranged widely - average duration of 15 to $120 \mathrm{~min} / \mathrm{session}, 2$ to 7 sessions/week, at an intensity of $40 \%$ of maximum heart to $85 \%$ of maximum oxygen uptake (V02 max) and over a duration of 24 to 52 weeks.

\section{Methodology of exercise training}

\section{Endurance aerobic training}

It as been the most popular choice since the first application in CHF patients. Patients exercised in a supervised environment three to five times a week, at an intensity calculated at $50-70 \% \mathrm{VO}_{2}$ peak or heart rate reserve or heart rate at peak exercise. Each session started with calisthenics for 10-15 minutes, followed by aerobic exercise such as walking on a treadmill or cycling on a stationary cycle ergometer for a total of $40 \mathrm{~min}$. Heart rate is monitored during each session, and blood pressure is measured electronically or manually at rest, during aerobic exercise and at the end of recovery. The duration of a training program averages 12 weeks, a duration sufficient to obtain improvements in functional capacity and quality of life. The results of the main trials using endurance protocols show increases in $\mathrm{VO}_{2}$ peak by $12-31 \%$.

\section{Interval training}

It is a methodology of training where exercise is divided in bouts of high and low intensity, starting with a low intensity (i.e. cycling at $30 \%$ of $\mathrm{VO}_{2}$ peak for $3 \mathrm{~min}$ ) followed by high intensity $\left(90 \%\right.$ of $\mathrm{VO}_{2}$ peak for 1 or $2 \mathrm{~min}$ ) for a total of $30 \mathrm{~min}$. This type of training has been recently emphasized by a group in Norway who exercised 27 patients (20 males, mean age 76 years) [11] with ischemic cardiomyopathy using high intensity interval training three times a week for 12 weeks as follows: 10 min warm up walking at $50-60 \%$ of $\mathrm{VO}_{2}$ peak, followed by 4-min intervals at $90-95 \%$ of peak heart rate, each interval separated by 3 -min active pauses, walking at $50-70 \%$ of peak heart rate [30]. The training session was terminated by a 3 -min cool-down at $50-70 \%$ of peak heart rate, with a total exercise time of 38 min per session. They compared 9 patients in the interval-training group with 9 patients in the endurance moderate training group and 9 untrained controls. The results were favorable to the interval training group in terms of improvements in $\mathrm{VO}_{2}$ peak $(+46 \%)$ and ejection fraction $(+35 \%)$. Major criticisms to this paper were the small number of subjects, the absence of follow up and the potential risk of arrhythmias due to the high intensity exercise.

Other studies followed with contrasting results. Of six studies comparing high intensity interval training with moderate intensity endurance training in stable HR-REF patients, 4 had similar improvements in $\mathrm{VO}_{2}$ peak, while 2 had no improvement in $\mathrm{VO}_{2}$ peak with endurance training, which is well below the average reported in the literature.

\section{Resistance training}

Resistance training (RT) is an accepted component of ExCR programs in CHF patients, because it enhances muscular strength and contributes to improved functional capacity. Conventional RT typically consists of lifting heavier weights with longer rest periods, whereas circuit training consists of lifting lighter weights with shorter rest periods between exercise. Circuit training is the most popular RT among CHF patients, with a greater aerobic component to workout and less intensity. In older women with CHF randomized to 10 weeks of RT or 
control, there was a $43 \%$ increase in muscle strength and a $49 \%$ increase in 6-min walk distance along with a $299 \%$ increase in submaximal endurance measured by the number of lifts at an intensity of $90 \%$ of baseline-1 repetition maximum. The beneficial effects of RT in CHF appear to be directed at improving skeletal muscle abnormalities and neuromuscular function, associated with an increased muscle mass, which correlates with $\mathrm{VO}_{2}$ peak [31]. Improvements in functional capacity has been mainly observed with RT in HF-REF patients, although RT has been included as an adjunctive modality to aerobic exercise training in the already cited Ex-DHF trial in patients with HF-PEF [32].

\section{Safety}

There is evidence that ExCR is safe in general, with no difference between patients with HF-REF or HF-PEF. In our database, we recorded 2 ventricular fibrillations over 28 years, which were promptly resolved with full recovery [33]. In the HF-ACTION Trial, of 2331 patients with HF-REF, 37 patients in the training group had at least 1 hospitalization due to an event that occurred during or within 3 hours after exercise, while 22 control patients had an adverse event despite no formal exercise program [21]. Only 1 patient had ICD discharge during exercise. No serious adverse training-related events have been reported in 6 trials that studied training in HFPEF patients (approximately 250 patients), which generally included older patients than in systolic CHF trials and a larger percentage of women [34].

\section{Benefits and mechanisms}

Ex-CR induces clear benefits to HF patients independent of age, sex and CHF type and etiology [35]. Benefits are related to improvement in functional capacity, which regresses in 2-3 weeks if patient stops exercising regularly. Recently, a 10-year supervised controlled endurance exercise program has been associated with a $32 \%$ relative risk reduction of cardiac mortality over 10 years, with a persistent maintenance of higher peak V02 and lower resting heart rate and ventilator efficiency in the training group [22]. In the ExTraMATCH meta-analysis on 801 HF-REF patients, there was a $35 \%$ reduction in mortality and hospital readmissions in trained patients versus untrained controls over a 2 -year follow up [9]. An analysis of $>600,000$ patients from the Medicare database addressed the effects of Ex-CR exercise training on mortality. Subgroup analyses of patients with CHF showed a 15\% lower mortality in CHF patients who participated in Ex-CR compared with carefully matched CHF patients who did not participate [36].

In a recent Cochrane review on 3647 patients, heart failure specific hospitalizations were reduced with exercise heart failure specific admissions (fixed effects RR $0.72,95 \%$ CI: 0.52 to $0.99 ; p=0.04$ ). Across the five studies that reported disease specific health-related quality of life using the Minnesota living with heart failure (MLWHF) questionnaire, there was a significant improvement in health-related quality of life (HRQoL) with exercise (random effects mean difference: -10.3, 95\% CI: -15.9 to $-4.8, \mathrm{p}=0.0003$ ). (37) Pooling across all studies reporting HRQoL regardless of outcome measure, there was also evidence of a significant improvement with exercise (random effects standardized mean difference: $-0.56,95 \% \mathrm{CI}$ : -0.82 to $-0.30, \mathrm{p}<0.0001$ ). No significant predictors of all-cause mortality or quality of life treatment response were found.

In HF-ACTION, exercise training-induced increases in $\mathrm{VO}_{2}$ peak were closely correlated with a better prognosis. Exercise training at 3 to 5 metabolic equivalent (MET) h/week was associated with $37 \%$ and $64 \%$ reductions in adjusted risks for death/hospitalization $(p=0.03)$ and cardiovascular death/heart failure hospitalization $(\mathrm{p}=0.001)$.
These results are in agreement with those showing that 1 MET increase in functional capacity is associated with a $12.4 \%$ lower mortality risk per year [38]. Moreover, for exercise to improve long-term outcomes, adherence to the exercise prescription is necessary [39].

The clinical benefits of exercise training in CHF can be expected to be long lasting. The effects of exercise training on prognosis may relate to its effects on autonomic function [40,41]. Neuro-humoral excitation and increased sympathetic nerve activity, both characteristic of CHF, are associated with long-term mortality, and exercise training reduces these abnormalities. The improvement in functional capacity is the results of central and peripheral adaptations induced by exercise training. Exercise training improves skeletal muscle aerobic capacity, as demonstrated by increased expression of oxidative enzymes such as citrate synthase and succinate dehydrogenase, an increased distribution of oxidative type I fibers and capillary to fiber, and an increased volume density of mitochondria which correlated with pre-post training $\mathrm{VO}_{2}$ peak $[42,43]$. The decreased sympathetic tone and the improved endothelial function are associated with enhanced vasodilatory capacity depending on greater nitric oxide biological activity and improved smooth muscle fibers relaxation. The improved endotheliumdependent relaxation is also related to decreased oxidative stress, as demonstrated by reduced skeletal muscle expression of NADPH oxidase and increased expression of ec-SOD dismutase [44-47]. Central adaptations have been described and may play a role to improve cardiocirculatory function. An anti-remodeling effect is associated with lower cardiac volumes and higher cardiac efficiency [48]. Diastolic relaxation is also improved after endurance training and translates into higher systolic efficiency [49]. Coronary adaptations have been also described, as greater vasodilatory capacity and improved collateral circulation, which correlates with improved myocardial perfusion and function $[50,51]$.

\section{Heart transplantation}

Exercise training is recommended after heart transplantation (HTx) because of positive effects on exercise performance and quality of life [52]. The majority of trials have used endurance aerobic exercise at moderate intensity. The American College of Sports Medicine and American Heart Association recommend exercising with intensity between $50 \%$ and $90 \%$ of maximum $\mathrm{VO}_{2}$, which refers to approximately $60 \%-95 \%$ of the maximum heart rate [53,54]. Accumulating evidence suggests that chronotropic incompetence is not a factor limiting exercise capacity in the majority of HTx recipients and that interval training is a feasible, safe and effective way to improve exercise capacity and general health in stable, long term HTx recipients. This type of exercise should be introduced and used more frequently among a broader audience. However, the transplanted heart seems to respond differently to this type of exercise, resulting mainly in peripheral improvements rather than improved cardiac function. The optimal timing to start a Ex-CR program is still a matter of debate. At present, there is not sufficient evidence to conclude that interval training is superior to endurance moderate exercise in HTx recipients.

\section{Conclusions}

In summary, Ex-CR is a therapeutic intervention able to induce clinical benefits related to improved functional capacity. The most important benefits are improved quality of life, reduced hospital readmissions and cardiac mortality. Exercise is an important component of CR, but patient's care is more complex requiring patient and family 
education, psychological support, diet counseling and drug optimization. The main objective of this multidisciplinary intervention is to correctly motivate patients to follow the CR program, which should be personalized according to individual clinical picture. The crucial point is the adherence to training, which is highly dependent on patient's motivation induced by cardiac rehabilitation team.

It seems that exercise is a trigger to stimulate protein synthesis by specific genes, which induce molecular adaptations able to maintain a higher aerobic capacity for months or years depending on the number of exercise sessions. It is crucial to maintain a specific stimulus by repeating exercise bouts at least 2-3 times per week all life long, since after 2-3 weeks of inactivity functional adaptations disappear.

\section{References}

1. Remes J, Reunanen A, Aromaa A, et al. Incidence of heart failure in eastern Finland: a population-based surveillance study. Eur Heart J 1992;13:588-92.

2. American Heart Association, 1999. Heart and Stroke Statistical Update. American Heart Association, Dallas.

3. Ho KKL, Anderson KM, Kannel WB, et al. Survival after the onset of congestive heart failure in Framingham Heart Study subject. Circulation 1993;88:107-15.

4. The SOLVD investigators. Effects of enalapril on survival in patients with reduced left ventricular ejection fraction and congestive heart failure. N Engl J Med 1991;325:293-302.

5. Pfeffer MA, Braunwald E, Moye LA, et al. Effect of captopril on mortality and morbidity in patients with left ventricular dysfunction after myocardial infarction: results of the Survival and Ventricular Enlargement Trial. N Engl J Med 1992;327:669-77.

6. Packer M, Bristow MR, Cohn JN, et al. The effect of carvedilol on morbidity and mortality in patients with chronic heart failure. $\mathrm{N}$ Engl J Med 1996;334:1349-55.

7. Leon AS, Barry A. Franklin B, et al. Cardiac Rehabilitation and Secondary Prevention of Coronary Heart Disease: An American Heart Association Scientific Statement From the Council on Clinical Cardiology (Subcommittee on Exercise, Cardiac Rehabilitation, and Prevention) and the Council on Nutrition, Physical Activity, and Metabolism Clinical Cardiology (Subcommittee on Physical Activity), in Collaboration With the American Association of Cardiovascular and Pulmonary Rehabilitation. Circulation 2005;111;369-76.

8. Perk J, De Cacker G, Gohlke H, et al. European Guidelines on cardiovascular disease prevention in clinical practice (ver. 2012). The Fifth Joint Task Force of the European Society of Cardiology and Other Societies on Cardiovascular Disease Prevention in Clinical Practice (constituted by representatives of nine societies and by invited experts) Developed with the special contribution of the European Association for Cardiovascular Prevention \& Rehabilitation (EACPR). Eur Heart J 2012;33:1635-701.

9. ExTraMATCH Collaborative Group. Exercise Training MetaAnalysis of Trials in patients with Chronic Heart failure. BMJ 2004;328:189-95

10. Alpert NR, Mulieri LA, Warshaw D. The failing human heart. Cardiovasc Res 2002;54:1-10.

11. Borlaug BA, Olson TP, Lam CSP, et al. Global cardiovascular reserve dysfunction in heart failure with preserved ejection fraction. J Am Coll Cardiol 2010;56:845-54.

12. Sullivan M, Higginbotham MB, Cobb FR. Exercise training in patients with severe left ventricular dysfunction. Circulation 1988;78: 06-15.

13. Coats AJS, Adamopoulos S, Meyer TE, et al. Effects of physical training in chronic heart failure. Lancet 1990; 335: 63-66.
14. Belardinelli R, Scocco V, Mazzanti M, et al. Effects of aerobic training in patients with moderate chronic heart failure. G Ital Cardiol 1992;22:919-30.

15. Jettè M, Heller R, Landry F, et al. Randomized four-week exercise program in patients with impaired left ventricular function. Circulation 1992;84:1561-7.

16. Hambrecht R, Niebauer J, Fiehn E, et al. G. Physical training in patients with stable chronic heart failure: effects on cardiorespiratory fitness and ultrastructural abnormalities of leg muscles. J Am Coll Cardiol 1995;25:1239-49.

17. Kavanagh T, Myers MG, Baigrie RS, et al. Quality of life and cardiorespiratory function in chronic heart failure: effects of 12 months' aerobic training. Heart 1996;76:42-6.

18. Keteyan S, Levine A, Brawner CA, et al. Exercise training in patients with heart failure: A randomized controlled trial. Ann Int Med 1996;124:1051-7.

19. Radaelli A, Coats AJS, Leuzzi S, et al. Physical training enhances sympathetic and parasympathetic control of heart rate and peripheral vessels in chronic heart failure. Clin Sci 1996;91:92-4.

20. Dubach P, Myers J, Dziekan G, et al. The effect of high intensity exercise training on central hemodynamic response to exercise in men with reduced left ventricular function. J Am Coll Cardiol 1997;29:1591-8.

21. O'Connor CM, Whellan DJ, Lee KL, et al. Efficacy and safety of exercise training in patients with chronic heart failure: HF-ACTION randomized controlled trial. JAMA 2009;301:1439-50.

22. Belardinelli R, Georgiou D, Cianci G, et al. 10-year exercise training in chronic heart failure. A Randomized controlled trial. J Am Coll Cardiol 2012;60:1521-8.

23. Edelmann F, Gelbrich G, Düngen H-D, et al. Exercise training improves exercise capacity and diastolic function in patients with heart failure with preserved ejection fraction: results of the ExDHF (Exercise training in Diastolic Heart Failure) pilot study. J Am Coll Cardiol 2011;58:1780-91.

24. Kitzman DW, Brubaker PH, Morgan TM, et al. Exercise training in older patients with heart failure and preserved ejection fraction. Circ Heart Fail 2010;3:659-67.

25. Smart N, Haluska B, Jeffriess L, et al. Exercise training in systolic and diastolic dysfunction: effects on cardiac function, functional capacity, and quality of life. Am Heart J 2007;153:530-6.

26. Gary RA, Sueta CA, Dougherty M, et al. Home-based exercise improves functional performance and quality of life in women with diastolic heart failure. Heart Lung 2004;33:210-8.

27. Haykowsky MJ, Brubaker PH, John JM, et al. Determinants of exercise intolerance in elderly heart failure patients with preserved ejection fraction. J Am Coll Cardiol 2011;58 265-74.

28. Alves AJ, Ribeiro F, Goldhammer E, et al. Exercise training improves diastolic function in heart failure patients. Med Sci Sports Exerc 2012;44:776-85.

29. Fujimoto N, Prasad A, Hastings JL, et al. Cardiovascular effects of 1 year of progressive endurance exercise training in patients with heart failure with preserved ejection fraction. Am Heart J 2012;164:869-77.

30. Wisloff U, Stoylen A, Loennechen JP, et al. Superior cardiovascular effect of aerobic interval training versus moderate continuous training in heart failure patients. A randomized study. Circulation 2007;115:3086-94.

31. Pu CT, Johnson MT, Forman DE, et al. Randomized trial of progressive resistance training to counteract the myopathy of chronic heart failure. J Appl Physiol 2001;90:2341-50.

32. Williams MA, Haskell WL, Ades PA, et al. Resistance exercise in individuals with and without cardiovascular disease: 2007 update: a scientific statement from the American Heart Association Council 
on Clinical Cardiology and Council on Nutrition, Physical Activity, and Metabolism. Circulation 2007;116:572-84.

33. Belardinelli R, Georgiou D, Cianci G, et al. Randomized, controlled trial of long-term moderate exercise training in chronic heart failure. Effects on functional capacity, quality of life, and clinical outcome. Circulation 1999;99:1173-82.

34. Taylor RS, Davies EJ, Dalal HM, et al. Effects of exercise training for heart failure with preserved ejection fraction: a systematic review and meta-analysis of comparative studies. Int $\mathbf{J}$ Cardiol 2012;162:6-13.

35. Pina IL, Apstein CS, Balady GJ, et al. Exercise and heart failure. A statement from the American Heart Association Committee on Exercise, Rehabilitation, and Prevention. Circulation 2003;107:1210-25.

36. Suaya JA, Stason WB, Ades PA, et al. Cardiac rehabilitation and survival in older coronary patients. J Am Coll Cardiol 2009;54:25-33.

37. Davies EJ, Moxham T, Rees K, et al. Exercise based rehabilitation for heart failure. Cochrane Database Syst Rev 2010;(4):CD003331.

38. Myers J, Prakash M, Froelicher V, et al. Exercise capacity and mortality among men referred for exercise testing. N Engl J Med 2002;346:793-801.

39. Ades PA, Keteyan SJ, Balady GJ, et al. Cardiac rehabilitation exercise and self care for chronic heart failure. JACC Heart Fail 2013;1:540-7.

40. Roveda F, Middlekauff HR, Rondon MU, et al. The effects of exercise training on sympathetic neural activation in advanced heart failure: a randomized controlled trial. J Am Coll Cardiol 2003;42:854-60.

41. Selig SE, Carey MF, Menzies DG, et al. Moderate-intensity resistance exercise training in patients with chronic heart failure improves strength, endurance, heart rate variability, and forearm blood flow. J Card Fail 2004;10:21-30.

42. Tyni-Lenne R, Gordon A, Jansson E, et al. Skeletal muscle endurance training improves peripheral oxidative capacity, exercise tolerance, and health-related quality of life in women with chronic congestive heart failure secondary to either ischemic cardiomyopathy or idiopathic dilated cardiomyopathy. Am J Cardiol 1997;80:1025-9.
43. Kiilavuori K, Naveri H, Salmi T, et al. The effect of physical training on skeletal muscle in patients with chronic heart failure. Eur J Heart Fail 2000;2:53-63.

44. Belardinelli R, Mucaj A, Lacalaprice F, et al. Coenzyme Q10 and exercise training in chronic heart failure. Eur Heart J 2006;27:2675-81.

45. Pina IL, Apstein CS, Balady GJ, et al. Exercise and heart failure: A statement from the American Heart Association Committee on Exercise, Rehabilitation and Prevention. Circulation 2003;107:1210-25.

46. Sullivan MJ, Green HJ, Cobb FR. Altered skeletal muscle metabolic responses to exercise in chronic heart failure: relation to skeletal muscle aerobic enzyme activity. Circulation 1991;84:1597-607.

47. Hambrecht R, Fiehn E, Weigl C, et al. Regular physical exercise corrects endothelial dysfunction and improves exercise capacity in patients with chronic heart failure. Circulation 1998;98:2709-15.

48. Giannuzzi P, Temporelli PL, Corra U, et al. Attenuation of unfavorable remodeling by exercise training in postinfarction patients with left ventricular dysfunction: results of the Exercise in Left Ventricular Dysfunction (ELVD) trial. Circulation 1997;96:1790-7.

49. Belardinelli R, Georgiou D, Cianci G, et al. Exercise training improves left ventricular diastolic filling in patients with dilated cardiomyopathy. Clinical and prognostic implications. Circulation 1995;91:2775-84.

50. Hambrecht R, Wolf A, Gielen S, et al. Effect of exercise on coronary endothelial function in patients with coronary artery disease. New Engl J Med 2000;342:454-60.

51. Belardinelli R, Georgiou D, Ginzton L, et al. Effects of moderate exercise training on thallium uptake and contractile response to low dose dobutamine of dysfunctional myocardium in patients with sichemic cardiomyopathy. Circulation 1998;97:553-61.

52. Nytroen K, Gullestad L. Exercise after heart transplantation: an overview. World J Transplant 2013;3:78-90.

53. American College of Sports Medicine, 2009. ACSM's guidelines for exercise testing and prescription, 8th ed. Wolters Kluwer, Lippincott Williams and Wilkins, Baltimore.

54. Fletcher GF, Balady GJ, Amsterdam EA, et al. Exercise standards for testing and training: a statement for healthcare professionals from the American Heart Association. Circulation 2001;104:1694-740. 